\title{
Excitation Retrieval of Microwave Linear Arrays from Phaseless Far Field Data
}

\author{
Benjamin Fuchs, Member, IEEE, and Laurent Le Coq
}

\begin{abstract}
A methodology to recover the excitations of microwave linear arrays from the measurements of far field magnitude only is proposed. The approach combines tools from convex optimization (to solve the phase retrieval problem) and a simple measurement procedure (to mitigate the non uniqueness of the solution). Numerical simulations in various representative and realistic configurations of noise and measurement sampling are presented and discussed. They show that it is possible to perfectly retrieve the array excitations from only the knowledge of far field magnitude by simply solving a convex optimization problem. In addition, the proposed approach, that only calls for readily available routines, is stable with respect to noise since the reconstruction performances degrades gracefully as the signal to noise ratio decreases.
\end{abstract}

Index Terms-Antenna measurements, phaseless measurement, phase retrieval, convex optimization.

\section{INTRODUCTION}

$\mathbf{M}$ ANY applications, in particular in imaging and optics, seek to reconstruct an object from the measurements of a signal. However, in many experimental setups, the phase of this signal is often difficult to measure accurately. The problem is then to reconstruct the object from magnitude measurements only. This inverse problem, known as phase retrieval, is frequently encountered in applied physics and engineering including X-ray and crystallography imaging [1], optics [2] and audio signal processing [3] to name just a few. A famous example that arises in a variety of fields is the one of recovering a signal from the magnitude of its Fourier transform.

The phase retrieval problem is also of uppermost interest in microwave antenna measurements where measuring accurately the phase may be costly and difficult in particular when dealing with high frequencies. Many efficient phaseless measurement methods have thus been proposed for antenna metrology [4][6], array diagnosis and imaging [7]-[9]. The advantages of such approaches are manifold. The probe positioning tolerances that is critical in near field measurement setups is relaxed. Moreover, magnitude-only measurements are by definition insensitive to potential instrument's phase drifts. Finally, phaseless measurements do not require the use of vector instruments whose cost is prohibitive as the frequency goes up.

Mathematically, the phase retrieval problem is notoriously very challenging.

On the one hand, it requires the development of efficient

Manuscript received xx, 2014; revised xx, xx.

The authors are with the IETR / University of Rennes I, France. (e-mail: \{benjamin.fuchs;laurent.le-coq\}@univ-rennes1.fr) and reliable reconstruction algorithms. A considerable amount of methods have been proposed in the literature. So far, most approaches were using alternating projection algorithms inspired by the seminal works of Gerchberg and Saxton [10] and Fienup [11]. These greedy techniques avoid an exhaustive search and they turn out to often work well in practice. However, these methods are not known to converge in general and they can stall in a local minima. Recently, phase retrieval problems have been treated using semidefinite relaxation and low-rank matrix recovery ideas [12]-[16]. To date, these convex approaches are the only ones providing guarantees on the recovery performances under some specific conditions (given in Section IV).

On the other hand, the phase retrieval problem may be illposed since its solution is, in general, not unique. In particular, the 1-D phase retrieval problem is known to admit multiple solutions [17]. Most existing methods seek to overcome this non uniqueness by imposing additional constraints on the signal to be retrieved such as its non-negativity or its sparsity. Recently, approaches assuming no prior information on the signal at all but using multiple structured measurements have been successfully proposed for optical setups [12], [18].

In this paper, we focus our investigation on the phase retrieval for microwave 1-D (linear) arrays. Our objective is to retrieve the complex array element excitations from its far field magnitude measurements. An efficient approach combining convex programming (to solve the phase retrieval problem) and two measurement runs (to mitigate the non uniqueness of the solution) is proposed. To the authors' best knowledge, it is the first time that convex relaxation based approaches are exploited to address phase retrieval problems in microwave applications. The goal of this paper is to empirically show that under appropriate conditions (of sampling and noise level), it is possible to uniquely recover the excitations of 1-D array from phaseless far field data.

The paper is organized as follows. The phase retrieval problem is formulated for 1-D microwave array imaging in Section II. Two algorithms to efficiently retrieve the array excitations are described and compared in Section III. Their application to array imaging problem and specifically a simple procedure to mitigate the solution ambiguities is proposed in Section IV. The numerical performances of the proposed phase retrieval procedure, namely the influence of the oversampling and the robutness to noise, are assessed in Section V. The conclusion and perspectives of this work are finally discussed. 


\section{Problem Formulation}

The phase retrieval seeks to reconstruct $N$ complex excitations $\boldsymbol{x}$ given only the magnitude of $M$ linear measurements $\boldsymbol{y}$. It can be formulated as follows:

$$
\text { find } \boldsymbol{x} \text { subject to }|\boldsymbol{A x}|=\boldsymbol{y}
$$

where $\boldsymbol{A} \in \mathbb{C}^{M \times N}$ is the sensing matrix, $\boldsymbol{x} \in \mathbb{C}^{N}$ and $\boldsymbol{y} \in \mathbb{R}^{M}$. This problem is difficult to solve because the set of real or complex numbers with a given magnitude is non-convex. An exhaustive search is untractable since, even in the real case, there are $2^{M}$ possible assignments of sign to the $M$ phaseless measurements. Moreover, the problem may be ill-posed. Depending on the properties of the matrix $\boldsymbol{A}$, the mapping from the magnitude measurements $\boldsymbol{y}$ to the excitations $\boldsymbol{x}$ is or is not one-to-one and hence unique recovery is not possible in general.

The geometry of the investigated problem, i.e. the imaging of a linear array, is represented in Fig. 1. The magnitude of

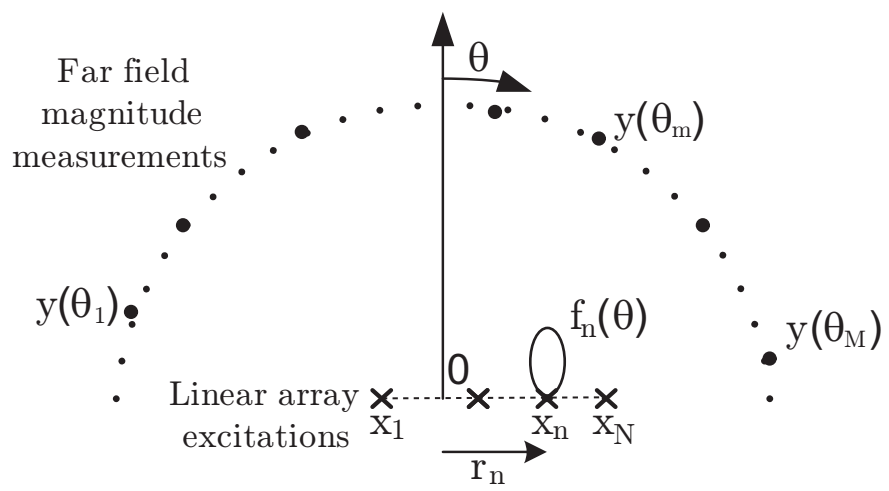

Fig. 1. Geometry of the linear array imaging problem with the notations.

the measured far field in the direction $\theta_{m}$ is related to the excitations $\boldsymbol{x}$ to be retrieved as follows:

$$
y\left(\theta_{m}\right)=\left|\sum_{n=1}^{N} \boldsymbol{A}_{m, n} x_{n}\right| \text { with } \boldsymbol{A}_{m, n}=f_{n}\left(\theta_{m}\right) e^{j \frac{2 \pi}{\lambda} r_{n} \sin \theta_{m}}
$$

where $\lambda$ is the free space wavelength, $r_{n}$ and $f_{n}\left(\theta_{m}\right)$ are the position and the far field pattern in the direction $\theta_{m}$ of the $n$-th antenna respectively and the time convention $e^{j \omega t}$ is omitted for clarity. Let us denote with $\boldsymbol{a}_{m}$ the vector of dimension $N$ whose elements are those of the $m$-th row of $\boldsymbol{A}$, it is the array steering vector in the direction $\theta_{m}$. The problem (1) can then be rewritten:

$$
\text { find } \boldsymbol{x} \text { subject to }\left|\boldsymbol{a}_{m}{ }^{T} \boldsymbol{x}\right|=y_{m}, \text { for } m=1, \ldots, M
$$

with $y_{m}=\boldsymbol{y}\left(\theta_{m}\right)$ and where $(.)^{T}$ is the transpose operator. The radiated field is thus related to the excitations by a discrete Fourier transform in the case of isotropic sources, i.e. when $f_{n}(\theta)=1, \forall n$. The problem is then ill-posed since many different sets of excitations have the same Fourier transform magnitude.

The ways (a) to solve the non-convex phase retrieval problem and (b) to mitigate the non-uniqueness (also called ambiguities) of the solution in order to uniquely recover the underlying excitations are addressed in Section III and IV respectively.

\section{Resolution Methods}

Two convex relaxation approaches are now described to approximate in order to efficiently solve the phase retrieval problem (2).

\section{A. PhaseLift}

The first convex relaxation of the phase retrieval problem has been introduced by Candes et al. [12]-[14]. He observed that the non-convex measurements $\boldsymbol{y}$ on vectors $\boldsymbol{x}$ become linear measurements on matrices $\boldsymbol{X}=\boldsymbol{x} \boldsymbol{x}^{H}$ where $(.)^{H}$ is the Hermitian conjugate. The measurements can be rewritten:

$$
y_{m}^{2}=\boldsymbol{x}^{H} \boldsymbol{A}_{m} \boldsymbol{x}=\operatorname{Tr}\left(\boldsymbol{A}_{m} \boldsymbol{X}\right)
$$

where $\boldsymbol{A}_{m}=\boldsymbol{a}_{m} \boldsymbol{a}_{m}{ }^{H}$ are hermitian matrices and $\boldsymbol{X}=\boldsymbol{x} \boldsymbol{x}^{H}$ is a rank-one Hermitian matrix.

The phase retrieval problem (2) becomes:

$$
\begin{array}{ll}
\text { find } & \boldsymbol{X} \\
\text { subject to } & \operatorname{Tr}\left(\boldsymbol{A}_{m} \boldsymbol{X}\right)=y_{m}^{2}, \quad m=1, \ldots, M \\
& \boldsymbol{X} \succeq 0 \\
& \operatorname{rank}(\boldsymbol{X})=1
\end{array}
$$

that is equivalent to:

$$
\begin{array}{ll}
\operatorname{minimize} & \operatorname{rank}(\boldsymbol{X}) \\
\text { subject to } & \operatorname{Tr}\left(\boldsymbol{A}_{m} \boldsymbol{X}\right)=y_{m}^{2}, \quad m=1, \ldots, M \\
& \boldsymbol{X} \succeq 0
\end{array}
$$

since there exists by definition a rank-one solution.

The problem (4) is a combinatorially hard problem. However, for positive semidefinite matrices, i.e. in this case since $\boldsymbol{X} \succeq 0$, the rank functional can be approximated by a convex surrogate, the trace norm as proposed in [19]. The problem (4) becomes:

$$
\begin{array}{ll}
\underset{\boldsymbol{X}}{\operatorname{minimize}} & \operatorname{Tr}(\boldsymbol{X}) \\
\text { subject to } & \operatorname{Tr}\left(\boldsymbol{A}_{m} \boldsymbol{X}\right)=y_{m}^{2}, \quad m=1, \ldots, M \\
& \boldsymbol{X} \succeq 0
\end{array}
$$

which is a semidefinite program that is then convex and efficiently solvable. The original vectorial phase retrieval problem is thus convexified by "lifting" it up to a matrix recovery problem hence the name PhaseLift introduced by [12], [13]. In practice, the measurements are contaminated by noise:

$$
y_{m}=\left|\boldsymbol{a}_{m}{ }^{T} \boldsymbol{x}+n_{m}\right|, \text { for } m=1, \cdots, M
$$

where $n_{m}$ is a noise term. The equalities in (5) no longer hold in presence of noise. The following formulation has then been proposed in [14]:

$$
\begin{aligned}
& \underset{\boldsymbol{X}}{\operatorname{minimize}} \sum_{m=1}^{M}\left|\operatorname{Tr}\left(\boldsymbol{A}_{m} \boldsymbol{X}\right)-y_{m}^{2}\right| \\
& \text { subject to } \boldsymbol{X} \succeq 0 .
\end{aligned}
$$

In words, solving (6) amounts to find the positive semidefinite matrix $\boldsymbol{X}$ that best fits the observed data in an $\ell_{1}$ sense. The 
solution of (6) will be from now on referred to as PL (for PhaseLift).

If the solution $\widehat{X}$ of (6) happens to have rank one, then $\widehat{\boldsymbol{X}}=\hat{\boldsymbol{x}} \hat{\boldsymbol{x}}^{H}$ and $\hat{\boldsymbol{x}}$ is the optimal solution of the original phase retrieval problem (2). Otherwise, one extracts the best rank one approximation of $\widehat{\boldsymbol{X}}: \widehat{\boldsymbol{X}}_{1}=\sigma_{1} \boldsymbol{u}_{1} \boldsymbol{u}_{1}{ }^{H}$ where $\sigma_{1}$ is the largest eigenvalue of $\widehat{\boldsymbol{X}}$ and $\boldsymbol{u}_{1}$ is the associated eigenvector. The vector $\hat{\boldsymbol{x}}=\sqrt{\sigma_{1}} \boldsymbol{u}_{1}$ is then an approximate solution of (2). For further information related to convex relaxations of quadratic problems, we refer the interested reader to the tutorial [20].

\section{B. PhaseCut}

A different strategy has been proposed in [15], [16] to solve the phase retrieval problem by explicitly separating the amplitude and phase variables in order to only optimize the value of the phase variables. By factoring out the magnitude information, we can write $\boldsymbol{A} \boldsymbol{x}=\operatorname{diag}(\boldsymbol{y}) \boldsymbol{u}$ where $\boldsymbol{u} \in \mathbb{C}^{M}$ is a phase vector satisfying $\left|u_{m}\right|=1$ for $m=1, \ldots, M$. The phase retrieval problem can be expressed as follows:

$$
\underset{\substack{\boldsymbol{u} \in \mathbb{C}^{M},\left|u_{m}\right|=1 \\ \boldsymbol{x} \in \mathbb{C}^{N}}}{\operatorname{minimize}}\|\boldsymbol{A} \boldsymbol{x}-\operatorname{diag}(\boldsymbol{y}) \boldsymbol{u}\|_{2}^{2}
$$

where we optimize over both variables $\boldsymbol{u}$ and $\boldsymbol{x}$.

The minimization over $\boldsymbol{x}$ is a standard least square and can be solved explicitly: $\boldsymbol{x}=\boldsymbol{A}^{\dagger} \operatorname{diag}(\boldsymbol{y}) \boldsymbol{u}$ where $\boldsymbol{A}^{\dagger}$ is the pseudo inverse of $\boldsymbol{A}$. The problem (7) is equivalent to:

$$
\underset{\boldsymbol{u} \in \mathbb{C}^{M},\left|u_{m}\right|=1}{\operatorname{minimize}}\left\|\boldsymbol{A} \boldsymbol{A}^{\dagger} \operatorname{diag}(\boldsymbol{y}) \boldsymbol{u}-\operatorname{diag}(\boldsymbol{y}) \boldsymbol{u}\right\|_{2}^{2} .
$$

The objective of (8) can be rewritten:

$$
\begin{aligned}
\left\|\boldsymbol{A} \boldsymbol{A}^{\dagger} \operatorname{diag}(\boldsymbol{y}) \boldsymbol{u}-\operatorname{diag}(\boldsymbol{y}) \boldsymbol{u}\right\|_{2}^{2} & =\left\|\left(\boldsymbol{A} \boldsymbol{A}^{\dagger}-\mathbf{I}\right) \operatorname{diag}(\boldsymbol{y}) \boldsymbol{u}\right\|_{2}^{2} \\
& =\boldsymbol{u}^{H} \operatorname{diag}\left(\boldsymbol{y}^{T}\right) \widetilde{\boldsymbol{R}} \operatorname{diag}(\boldsymbol{y}) \boldsymbol{u}
\end{aligned}
$$

where $\widetilde{\boldsymbol{R}}=\left(\boldsymbol{A} \boldsymbol{A}^{\dagger}-\mathbf{I}\right)^{H}\left(\boldsymbol{A} \boldsymbol{A}^{\dagger}-\mathbf{I}\right)=\left(\mathbf{I}-\boldsymbol{A} \boldsymbol{A}^{\dagger}\right)$. The phase retrieval problem (1) becomes:

$$
\begin{aligned}
& \underset{\boldsymbol{u} \in \mathbb{C}^{M}}{\operatorname{minimize}} \boldsymbol{u}^{H} \boldsymbol{R} \boldsymbol{u} \\
& \text { subject to }\left|u_{m}\right|=1, \quad m=1, \ldots, M
\end{aligned}
$$

where the Hermitian matrix $\boldsymbol{R}=\operatorname{diag}(\boldsymbol{y})\left(\mathbf{I}-\boldsymbol{A} \boldsymbol{A}^{\dagger}\right) \operatorname{diag}(\boldsymbol{y})$ is positive semidefinite.

The problem (9) is equivalent to:

$$
\begin{array}{cl}
\underset{\boldsymbol{U}}{\operatorname{minimize}} & \operatorname{Tr}(\boldsymbol{R} \boldsymbol{U}) \\
\text { subject to } & \operatorname{diag}(\boldsymbol{U})=1 \\
& \boldsymbol{U} \succeq 0 \\
& \operatorname{rank}(\boldsymbol{U})=1 .
\end{array}
$$

By dropping the non-convex rank constraint, we obtain the following convex relaxation:

$$
\begin{array}{ll}
\underset{\boldsymbol{U}}{\operatorname{minimize}} & \operatorname{Tr}(\boldsymbol{R} \boldsymbol{U}) \\
\text { subject to } & \operatorname{diag}(\boldsymbol{U})=1 \\
& \boldsymbol{U} \succeq 0
\end{array}
$$

that is very similar to the MaxCut semidefinite program hence the name PhaseCut (denoted PC in the sequel).
As in the PL procedure, if the solution has a rank larger than one, the leading eigenvector of $\boldsymbol{U}$ is used to build an approximate solution $\hat{\boldsymbol{u}}$ of (11) from which an approximate solution of (2) is derived: $\hat{\boldsymbol{x}}=\boldsymbol{A}^{\dagger} \operatorname{diag}(\boldsymbol{y}) \hat{\boldsymbol{u}}$.

\section{Discussion}

By trading non-convex constraints into convex ones, we have transformed a vector problem into a matrix one. This "lifting" leads to a much larger representation of the state space which means a higher computational cost. It also implies that we must deal with a highly underdetermined problem since for the PL procedure, there are $N^{2}$ unknowns (instead of only $N$ ) for $M=O(N)$ magnitude measurements. As explained in [21], this apparent lack of data is compensated by the fact that we are only looking for rank one matrices. It is also worth noting that the computational complexity of the PC algorithm (11) is higher than the one of PL (6) since we are looking in the space of Hermitian matrices of dimension $M$ for PC instead of only $N$ for PL.

Last but not least, both convex relaxations (6) and (11) can be solved optimally by readily available software such as CVX [22].

\section{Applichtion to Array Imaging Problems}

The problem of ambiguity in phase retrieval problems is first discussed, existing solutions to mitigate this non uniqueness are reviewed and a simple approach suitable to microwave antenna arrays is proposed. Then, the way to quantify the recovery performances of the proposed approach are detailed.

\section{A. Non-uniqueness (Ambiguities) of the solution}

In the general case, the solution to the phase retrieval problem (1) is not unique and the best we can strive for is to retrieve $x_{n}$ up to a phase $\alpha$, a "mirror function", and an unknown shift $\delta$. Indeed, if $x_{n}$ with $n=1, \ldots, N$ is a solution to (1), then $e^{j \alpha} x_{n}, x_{N+1-n}^{*}$ and $x_{n}$ at locations $r_{n}-\delta$ are respectively also solution. Note that $a^{*}$ is the complex conjugate of $a$. Since we assume that the positions $r_{n}$ of the array elements are known, the last ambiguity (unknown shift) type does not exist. More specifically, for a linear array composed of $N$ equispaced identical elements, there are up to $2^{N-1}$ possible solutions as explained in [23].

To mitigate the ambiguities arising in phase retrieval problems, the oversampling in the Fourier domain (i.e. the increasing of the measurement points $M$ ) has been shown to almost always work in the case of 2D Fourier transform [24]. However, this technique offers no benefits for 1D recovery as discussed in [12], [15], [17] and therefore cannot be used for our application.

Another approach is to try to render the sensing matrix $\boldsymbol{A}$ as "random" as possible in order to make unique recovery possible. For that purpose, a measurement setup using of an electronically controlled reflectarray between the microwave antenna under test and the measurement probe has been proposed in [25]. The idea is to switch randomly the elements of the reflectarray to obtain a random sampling of the field 
radiated by the antenna under test. This solution, that is theoretically very appealing, seems in practice difficult and costly to implement.

In optics, many techniques have been successfully applied to resolve the phase ambiguity problem. They consist in using multiple independent measurements to somehow gather different views of the object under test as reviewed in [12]. Thus, taking multiple diffraction patterns by adding a mask or modulating the light beam with an optical grating usually yields uniqueness. In that case, the sensing matrix can be seen as the product between the discrete Fourier transform matrix and a mask or filter that is a diagonal matrix with either random binary or Gaussian entries.

Recently, an interesting framework [26] (called vectorial phase retrieval by the authors) has been proposed to efficiently solve the phase retrieval problem. It is shown that the unique reconstruction of two signals is possible by measuring their spectral intensity signals and their pairwise interferences.

The previously described techniques are difficult to implement in microwaves that is why we propose the following approach. As done in holography [27], we assume that we have a reference antenna whose complex radiation pattern $y^{r e f}(\theta)$ (the vector $\boldsymbol{y}^{\text {ref }}$ of dimension $M$ after discretization) is known. We measure the magnitude of the far field radiated by:

- the array under test $\boldsymbol{y}^{A U T}=\left|\boldsymbol{A}^{A U T} \boldsymbol{x}\right|$ and

- the interference between the array under test and the reference antenna $\boldsymbol{y}^{A U T+r e f}=\left|\boldsymbol{A}^{A U T} \boldsymbol{x}+\alpha \boldsymbol{y}^{\text {ref }}\right|$

where $\alpha$ is the excitation (not necessarily known) of the reference antenna.

This simple procedure allows to mitigate the ambiguities due to the "mirror function" and the excitations are then determined up a global phase. If in addition the phase of the excitation $\alpha$ is known (after a calibration for instance), then the solution $\boldsymbol{x}$ is unique.

The matrix $\boldsymbol{A} \in \mathbb{C}^{2 M \times(N+1)}$ and the measurement vector $\boldsymbol{y}$ in (1) becomes:

$$
\boldsymbol{A}=\left[\begin{array}{l|l}
\boldsymbol{A}^{A U T} & \mathbf{0}_{M} \\
\boldsymbol{A}^{A U T} & \boldsymbol{y}^{\text {ref }}
\end{array}\right] \text { and } \boldsymbol{y}=\left[\begin{array}{l}
\boldsymbol{y}^{A U T} \\
\boldsymbol{y}^{A U T+r e f}
\end{array}\right]
$$

where $\mathbf{0}_{M}$ is a vector of zeros of dimension $M$.

\section{B. Recovery Performances}

1) Recovery Error: The phase retrieval algorithms (6) and (11) compute an approximate solution $\hat{\boldsymbol{x}}$ from $\boldsymbol{y}=|\boldsymbol{A} \boldsymbol{x}|$. Their performances are typically assessed by computing the relative "distance" between the exact solution $\boldsymbol{x}$ and the recovered one $\hat{\boldsymbol{x}}$. Special care must be taken since a solution may be unique up to a global phase (for complex values) / sign (for real values) (if the excitation of the reference antenna is not known). Thus, the error in excitation denoted $\epsilon(\boldsymbol{x}, \hat{\boldsymbol{x}})$ can be computed as:

$$
\min _{\alpha \in[0,2 \pi]} \frac{\left\|e^{j \alpha} \boldsymbol{x}-\hat{\boldsymbol{x}}\right\|_{2}}{\|\boldsymbol{x}\|_{2}} \text { or } \frac{\left\|\boldsymbol{x} \boldsymbol{x}^{H}-\hat{\boldsymbol{x}} \hat{\boldsymbol{x}}^{H}\right\|_{F}}{\left\|\boldsymbol{x} \boldsymbol{x}^{H}\right\|_{F}}
$$

where $\|\cdot\|_{F}$ stands for the Frobenius norm. This second definition, easier to compute, will be used in Section V.
The error over the measured field amplitudes is:

$$
\epsilon(|\boldsymbol{A} \boldsymbol{x}|,|\boldsymbol{A} \hat{\boldsymbol{x}}|)=\frac{\||\boldsymbol{A} \boldsymbol{x}|-|\boldsymbol{A} \hat{\boldsymbol{x}}|\|_{2}}{\|\boldsymbol{A} \boldsymbol{x}\|_{2}} .
$$

If a low excitation error $\epsilon(\boldsymbol{x}, \hat{\boldsymbol{x}})$ implies a low error over the measured field amplitudes $\epsilon(|\boldsymbol{A} \boldsymbol{x}|,|\boldsymbol{A} \hat{\boldsymbol{x}}|)$, the opposite is not necessarily true. Indeed, when the phase retrieval problem does not have a unique solution, we may have a low $\epsilon(|\boldsymbol{A} \boldsymbol{x}|,|\boldsymbol{A} \hat{\boldsymbol{x}}|)$ but $\epsilon(\boldsymbol{x}, \hat{\boldsymbol{x}}) \gg \epsilon(|\boldsymbol{A x}|,|\boldsymbol{A} \hat{\boldsymbol{x}}|)$.

Although the quantities (13) and (14) are useful to assess and compare the efficiency of the algorithms PL and PC, they are not very speaking. In order to appreciate the results with more physical insights, we compute the mean values of the excitation amplitude ratio and phase difference (denoted $\mu_{m}(\boldsymbol{x}, \hat{\boldsymbol{x}})$ and $\mu_{p}(\boldsymbol{x}, \hat{\boldsymbol{x}})$ respectively):

$$
\begin{aligned}
& \mu_{m}(\boldsymbol{x}, \hat{\boldsymbol{x}})=\frac{1}{N} \sum_{i=1}^{N} \delta_{m i} \text { with } \delta_{m i}=\frac{\left|\hat{\boldsymbol{x}}_{i}\right|}{\left|\boldsymbol{x}_{i}\right|} \\
& \mu_{p}(\boldsymbol{x}, \hat{\boldsymbol{x}})=\frac{1}{N} \sum_{i=1}^{N}\left|\delta_{p i}\right| \text { with } \delta_{p i}=\left(\angle \hat{\boldsymbol{x}}_{i}-\Delta \phi\right)-\angle \boldsymbol{x}_{i}
\end{aligned}
$$

where $\Delta \phi$ the global phase shift between $\boldsymbol{x}$ and $\hat{\boldsymbol{x}}$ is equal to $\Delta \phi=\frac{1}{N} \sum_{i=1}^{N}\left(\angle \hat{\boldsymbol{x}}_{i}-\angle \boldsymbol{x}_{i}\right)$.

We derive from (15) the standard deviation in amplitude and phase $\left(\sigma_{m}(\boldsymbol{x}, \hat{\boldsymbol{x}})\right.$ and $\sigma_{p}(\boldsymbol{x}, \hat{\boldsymbol{x}})$ respectively):

$$
\begin{aligned}
& \sigma_{m}(\boldsymbol{x}, \hat{\boldsymbol{x}})=\left[\frac{1}{N} \sum_{i=1}^{N}\left(\delta_{m i}-\mu_{m}(\boldsymbol{x}, \hat{\boldsymbol{x}})\right)^{2}\right]^{1 / 2} \\
& \sigma_{p}(\boldsymbol{x}, \hat{\boldsymbol{x}})=\left[\frac{1}{N} \sum_{i=1}^{N}\left(\delta_{p i}-\mu_{p}(\boldsymbol{x}, \hat{\boldsymbol{x}})\right)^{2}\right]^{1 / 2}
\end{aligned}
$$

in order to measure the degree of confidence in the retrieved excitation magnitudes and phases.

2) Noise: The reconstruction of the excitations in presence of noise is crucial for practical applications. In our experiments, the magnitude measurement $y_{m}$ is polluted by a Gaussian white noise $n_{m}$ as follows: $y_{m}=\left|\boldsymbol{a}_{m}^{T} \boldsymbol{x}+n_{m}\right|$. The level of this noise is quantified by the Signal-to-Noise Ratio (SNR): $\operatorname{SNR}_{d B}=10 \log _{10}\left(P_{\text {signal }} / P_{\text {noise }}\right)$ where $P_{\text {signal }}=$ $\max _{m=1, \cdots, M}\left(\left|\boldsymbol{a}_{m}{ }^{T} \boldsymbol{x}\right|^{2}\right)$ is the maximum measured power. In order to estimate the SNR of a far field measurement in an anechoic chamber, both the full equipment system (noise floor of the receiver, transmitted power and losses due to the cables and rotary joints) and the reflectivity of the chamber itself must be taken into account. A SNR value of $60 \mathrm{~dB}$, that corresponds to a reasonably good anechoic chamber, will be considered for the numerical applications of Section V.

3) Sampling $(M / N)$ : Up to date, the convex approaches are the only ones to offer guarantees on the recovery performances for phase retrieval problems. It means that under appropriate conditions, the difficult problem (2) is equivalent to the convex program (6) or (11) in the sense that they have the same unique solution. If the number $M$ of equations $\left|\boldsymbol{a}_{m}{ }^{T} \boldsymbol{x}\right|=y_{m}$ are sufficiently randomized and at least on the order of $N$, it has been demonstrated in [14] that the solution to the convex relaxation (6) is exact (i.e. it is the same as the one of (2)) with a probability approaching one exponentially, as $M$ is 
increased. It is important to point out that these results have been established for sensing matrices $\boldsymbol{A}$ with Gaussian i.i.d. (independent and identically distributed) entries.

In the next Section, we will numerically check if similar results hold in the case of 1-D microwave array imaging, i.e. when the sensing matrix is not Gaussian but of the form of a discrete Fourier Transform.

\section{NUMERICAL RESUlts}

The goal of this Section is to numerically assess the recovery performances of the phase retrieval algorithm (6) and (11). In the retrieval procedure, the element positions and radiation patterns of the array under test, i.e. of the sensing matrix $\boldsymbol{A}$, and the reference antenna $y^{r e f}$ are assumed known.

For each configuration, the simulation is repeated 100 times with different random excitations (whose magnitude and phase are chosen uniformly between $[0,1]$ and $[0,2 \pi]$ respectively), different random measurement vectors (whose angle are chosen uniformly such that $-1 \leq \sin \theta \leq 1$ ) and a Gaussian white noise in order to get meaningful results. All presented results are average values over these 100 simulations.

\section{A. Linear Array of Isotropic Sources}

1) Settings: We consider a linear array composed of $N$ isotropic half wavelength spaced elements that are excited by a random amplitude and phase $x_{n}$. A reference antenna (also isotropic) with an arbitrary excitation (fixed but chosen randomly in the simulations) is placed at an arbitrary distance ( $2 \lambda$ in the simulations) from the extremity of the array under test. It has been checked numerically that both the position and excitation value of this source with respect to the array under test do not impact the recovery performances.

2) Influence of the Sampling: The influence of the ratio $M / N$ (number of measurement points over number of excitations to retrieve) on the recovery performances is investigated. The error in excitations $\epsilon(\boldsymbol{x}, \hat{\boldsymbol{x}})$ is plotted as a function of the sampling for linear arrays of various elements $N$ in Fig. 2. In absence of noise, there is a clear transition between 'bad' and 'good' recovery at $M=2 N$. In presence of a realistic noise $(\mathrm{SNR}=60 \mathrm{~dB})$, a less sharp transition also exists around $M=2 N$. Generally speaking, for a given sampling $M / N$, the recovery performances are better for a small number of array elements $N$.
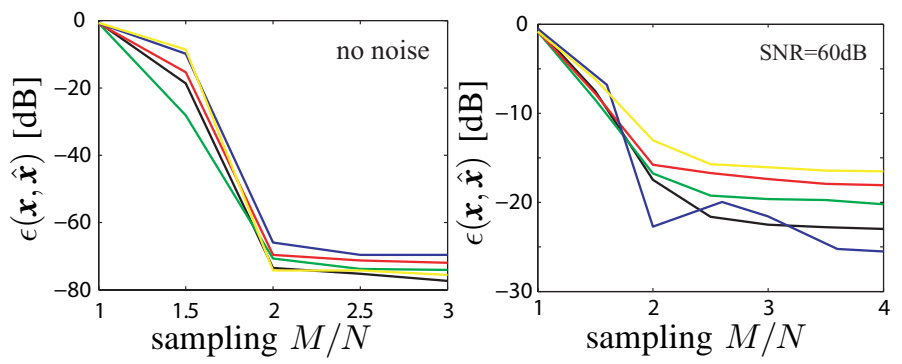

Fig. 2. Error in the retrieved excitation amplitudes as a function of the sampling with PhaseLift for linear array of various elements $(N=5,10,20$, $30,40)$.
To evaluate the reconstruction quality as a function of the sampling, let us plot in Fig. 3 the reconstruction errors in amplitude and phase for a linear array of $N=10$ elements in presence of noise $(\mathrm{SNR}=60 \mathrm{~dB})$. We retrieve, as seen above, the significant improvement in recovery performances when $M=2 N$. Then, The oversampling helps to further improve up to a certain extent the recovery performances as shown in Fig. 3 for $M>2 N$.
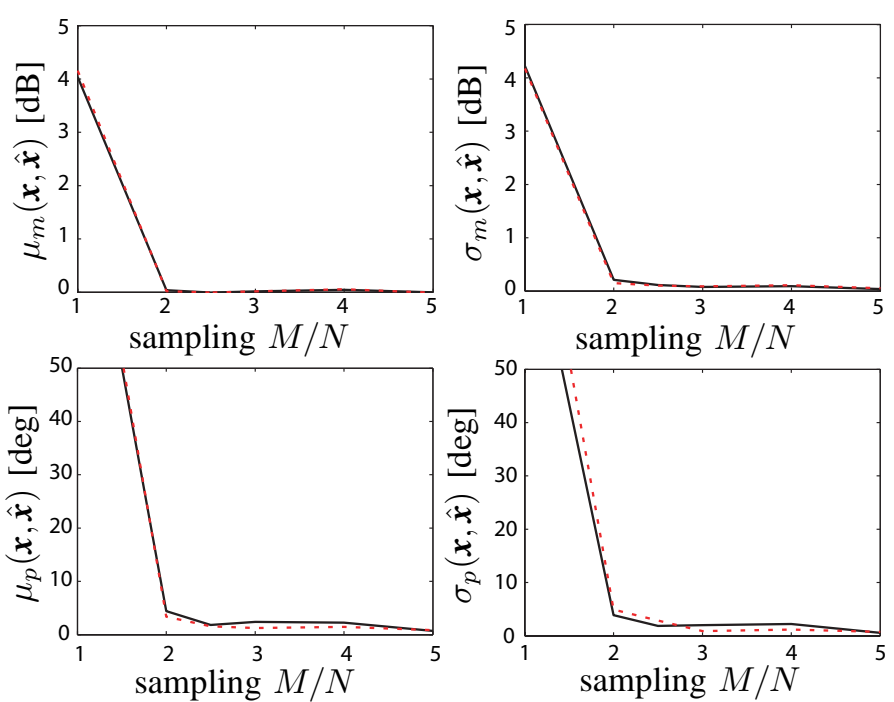

Fig. 3. Recovery performances with PhaseLift (solid line) and PhaseCut (dotted line) for a linear array of $N=10$ isotropic sources: influence of the sampling $(M / N)$ for measurements with a SNR of $60 \mathrm{~dB}$.

3) Robustness to Noise: Let us choose a sampling $M=2 \mathrm{~N}$ in order to see the influence of the noise on the excitation recovery.

The influence of the SNR on the retrieved excitations and field amplitudes $(\epsilon(\boldsymbol{x}, \hat{\boldsymbol{x}})$ and $\epsilon(|\boldsymbol{A} \boldsymbol{x}|,|\boldsymbol{A} \hat{\boldsymbol{x}}|)$ respectively) is plotted in Fig. 4. There is clearly a linear behavior between these errors and the SNR with a log-log scale. This graceful degradation shows that both convex approaches PL and PC provide a stable recovery in presence of noise.

The reconstruction errors in excitation amplitude and phase
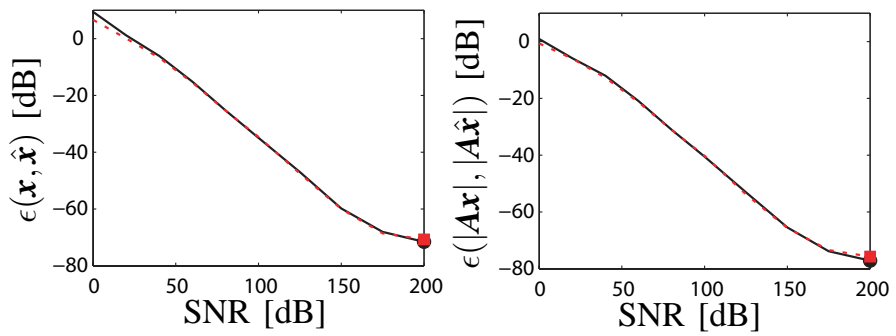

Fig. 4. Error in the retrieved excitations and field amplitudes for a linear array of $N=10$ elements as a function of the SNR with PhaseLift (solid line) and PhaseCut (dotted line). The markers (circle for PL and square for PC) are the results without noise.

are plotted in Fig. 5 as a function of the SNR. They show that above a given SNR (of $60 \mathrm{~dB}$ for $M / N=2$ ), the reconstruction is very good in amplitude and fairly good in phase. In presence of important noise $(\mathrm{SNR}<60 \mathrm{~dB})$, the algorithm 
PC seems to yield a better reconstruction (especially in phase) than PL. This better performance may be explained by the decoupling between magnitude and phase reconstruction of the PC procedure (see Section III-B).
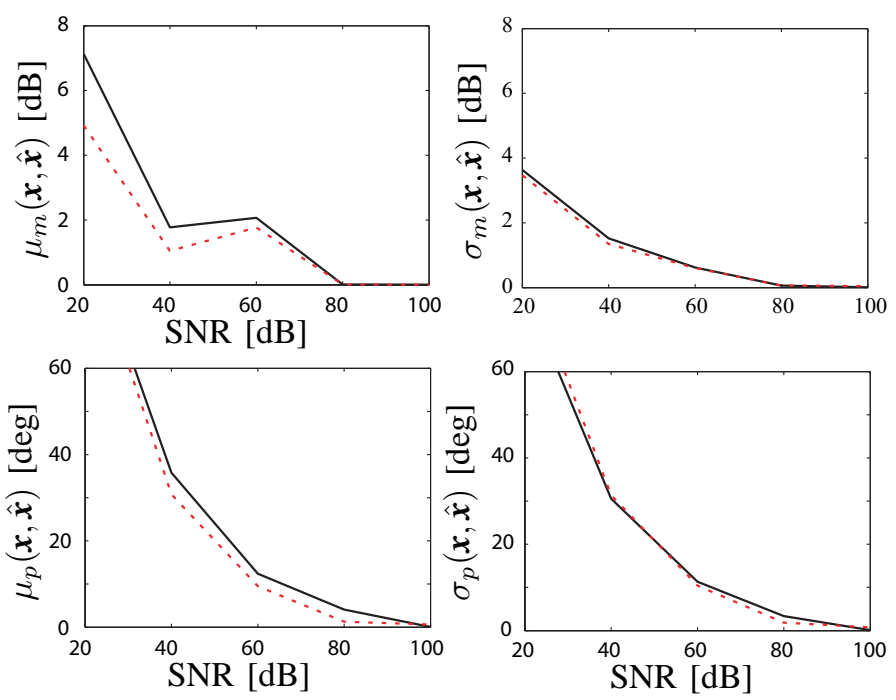

Fig. 5. Recovery performances for a linear array of $N=10$ isotropic sources with a sampling $M=2 N$ : influence of the SNR with PhaseLift (solid line) and PhaseCut (dotted line).

\section{B. Linear Array of Realistic Sources}

1) Settings: Let us consider a linear array of five patches with an open ended waveguide that plays the role of the antenna of reference to overcome the ambiguity problem, see Fig. 6. The field radiated by the array is computed by a 3D full wave commercial software (Ansys HFSS) in order to get the sensing matrix $\boldsymbol{A}$ and far field magnitude $\boldsymbol{y}$.

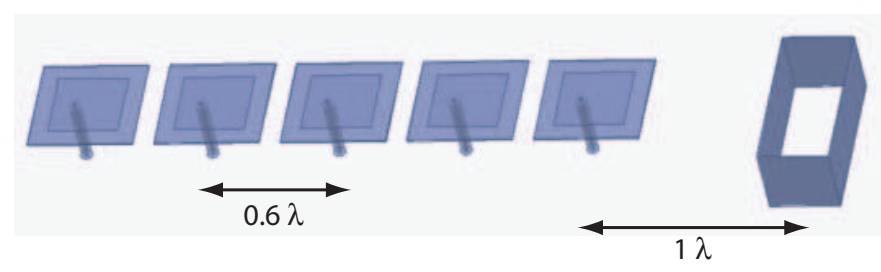

Fig. 6. View of the array of five patchs whose excitations are to be retrieved with the reference antenna (an open ended waveguide) to overcome the ambiguity problem.

2) Influence of the Sampling: In absence of noise, a perfect recovery, i.e. an error in excitation amplitude and phase lower than $10^{-5} \mathrm{~dB}$ and deg respectively and a very low standard deviation, is achieved as soon as $M \geq 2 N$. Generally speaking, better recovery performances are achieved in the case of realistic sources compared to isotropic ones. The ambiguity problem is indeed intrinsically reduced when the radiation patterns of each element are not all identical.

The influence of the oversampling on the recovery performances in presence of noise $(\mathrm{SNR}=60 \mathrm{~dB})$ are plotted in Fig. 7. An oversampling $M>6 N$ does not bring any further improvement.
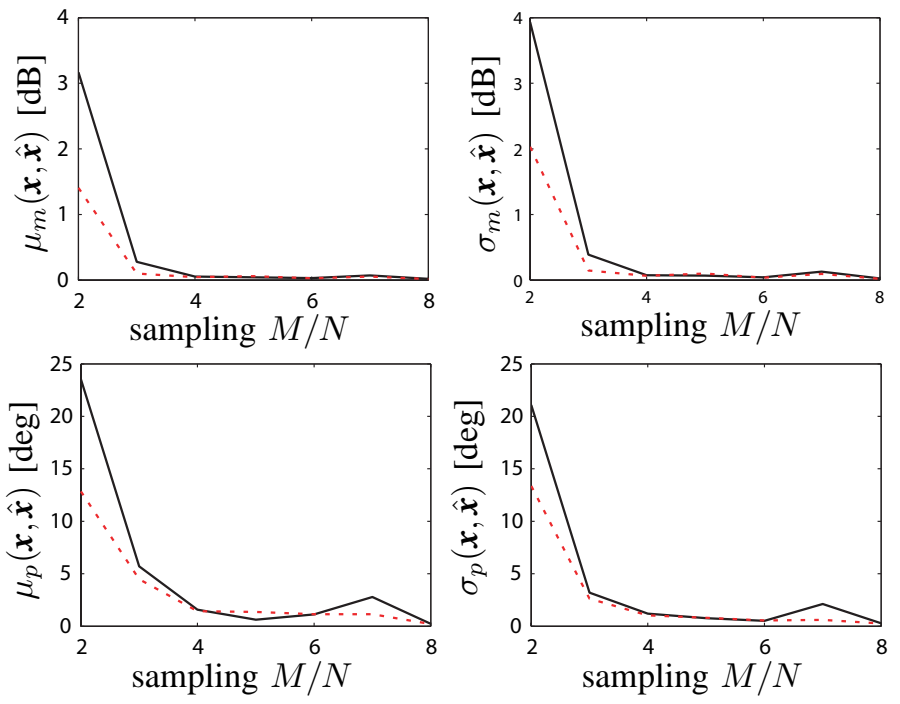

Fig. 7. Recovery performances for a linear array of five patches: influence of the sampling $(M / N)$ for measurements with a SNR of $60 \mathrm{~dB}$ with PhaseLift (solid line) and PhaseCut (dotted line).

3) Robustness to Noise: The error in the retrieved excitations and field amplitudes increases linearly (in a log-log scale) with the noise. This robustness to the noise (not reported) is similar to the one of isotropic sources of Section V-A.3.

The reconstruction errors in excitation magnitudes and phases are shown in Fig. 8 as a function of the SNR for an oversampling of $M=3 N$. In that case, a very good recovery is achieved for SNRs greater than $60 \mathrm{~dB}$. The algorithm PC provides a slightly better recovery in case of low SNR as for the case of isotropic sources (see Section V-A.3).
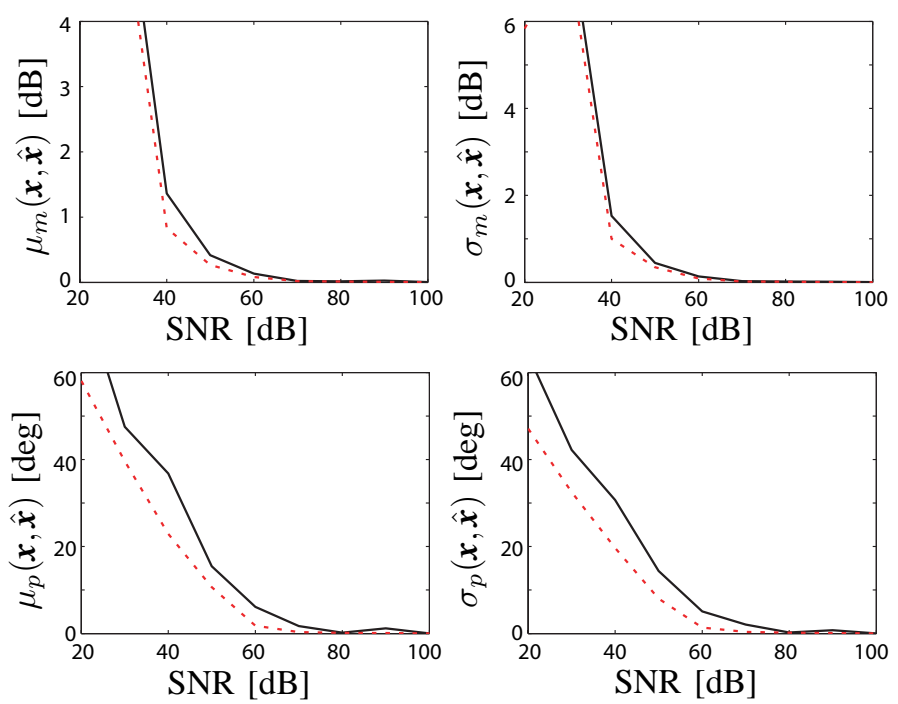

Fig. 8. Recovery performances for a linear array of five patches: influence of the SNR with PhaseLift (solid line) and PhaseCut (dotted line) for a sampling $M=3 N$.

\section{CONCLUSION}

A methodology has been proposed to efficiently solve the phase retrieval problem arising in microwave linear array. The 
goal is to retrieve the complex array excitations from the measurements of the far field magnitude only. A procedure combining convex optimization and two measurement runs has been developed and numerically assessed in various representative and realistic conditions of noise and sampling. Promising results have been obtained: an excellent amplitude and phase excitation recovery is achieved for a sampling (number of measurement samples over excitation points) of above a factor two in presence of a realistic noise of SNR equal to $60 \mathrm{~dB}$. This procedure is new in the field of microwave antenna measurements and seems to be promising to significantly reduce the cost of future microwave imaging systems.

This work calls for several appealing extensions. First, the application of the proposed approach to 2-D array phase retrieval should be straightforward all the more as the problem of ambiguities can be directly overcome by oversampling. The effort should then focus on reducing the computational complexity to solve the convex relaxations. Second, it would be of great interest to assess the efficiency of the proposed phase retrieval approach in the case of near field data. The proposed approach for far field data could be a priori used by only changing the sensing matrix. However, several crucial parameters such as the distance array under test - probe and the range of the probe measurements have to be carefully addressed. Finally, the phase retrieval method must be tested on real measurement data and we hope to report such experiment in a future publication.

\section{REFERENCES}

[1] R.W. Harrison "Phase problem in crystallography," J. Opt. Soc. Am., vol. 10, no. 5, pp. 1045-1055, 1993.

[2] A. Walther "The question of phase retrieval in optics," Opt. Acta, vol. 10, pp. 41-49, 1963.

[3] D. Griffin and J. Lim, "Signal estimation from modified short-time Fourier transform," IEEE Trans. Acoust. Speech Sig. Proc., vol. 32, no. 2, pp. 236-243, 1984.

[4] O.M. Bucci, G. D'Elia, G. Leone, and R. Pierri, "Far-Field Pattern Determination from the Near-Field Amplitude on Two Surfaces," IEEE Trans. Antennas Propag., vol. 38, no. 11, pp. 1772-1779, May 1990.

[5] T. Isernia, G. Leone, and R. Pierri, "Radiation Pattern Evaluation from Near-field Intensities on Planes," IEEE Trans. Antennas Propag., vol. 44, no. 5, pp. 701-710, May 1996.

[6] M.D. Migliore, F. Soldovieri, and R. Pierri, "Far-field antenna pattern estimation from near-field data using a low-cost amplitude-only measurement setup,” IEEE Trans. Instrum. Meas., vol. 49, no. 1, pp. 71-76, Feb. 2000.

[7] D. Morris, "Phase retrieval in the radio holography of reflector antennas and radio telescopes," IEEE Trans. Antennas Propag., vol. 33, pp. 749755, July 1985.

[8] R.G. Yaccarino and Y. Rahmat-Samii, "Phaseless Bi-Polar Planar NearField Measurements and Diagnostics of Array Antennas," IEEE Transactions on Antennas and Propagation, vol. 47, no. 3, pp. 574-583, March 1999.

[9] O.M. Bucci, A. Capozzoli, and G. D'Elia, "Diagnosis of Array Faults from Far-Field Amplitude-Only Data," IEEE Trans. Antennas Propag., vol. 48, no. 5, pp. 647-652, May 2000.

[10] R. Gerchberg and W. Saxton, "A practical algorithm for the determination of phase from image and diffraction plane pictures," Optik, vol. 35, pp. 237-246, 1972.

[11] J. Fienup, "Phase retrieval algorithms: a comparison," Applied Optics, vol. 21 , no. 15 , pp. 2758-2769, 1982.

[12] E.J. Candes, Y.C. Eldar, T. Strohmer, and V. Voroninski. "Phase retrieval via matrix completion," SIAM J. on Imaging Sciences, no. 6, vol. 1, pp. 199-225, 2011.
[13] E.J. Candes, T. Strohmer, and V. Voroninski. "Phaselift : exact and stable signal recovery from magnitude measurements via convex programming," Commun. Pure Appl. Math., no. 66, vol. 8, pp. 1241-1274, 2011.

[14] E.J. Candès and X. Li, "Solving quadratic equations via PhaseLift when there are about as many equations as unknowns," Foundations of Computational Mathematics, 2013.

[15] I. Waldspurger, A. d'Aspremont, and S. Mallat "Phase Recovery, MaxCut and Complex Semidefinite Programming," Preprint on ArXiv: 1206.0102. (2013).

[16] F. Fogel, I. Waldspurger, A. d'Aspremont, "Phase Retrieval for Imaging Problems," Preprint on ArXiv: 1304.7735 (2013).

[17] Y. Bruck and L. Sodin, "On the ambiguity of the image reconstruction problem," Opt. Commun., vol. 30, no. 3, pp. 304-308, 1979.

[18] E. Candes, X. Li, and M. Soltanolkotabi, "Phase Retrieval from masked Fourier transforms," Preprint on ArXiv: 1310.3240 (2013).

[19] M. Fazel, H. Hindi, and S. Boyd, "Rank Minimization and Applications in System Theory," Proc. American Control Conference, Boston, Massachusetts, June 2004.

[20] Z.-Q. Luo, W.-K. Ma, A. Man-Cho So, Y. Ye, and S. Zhang, "Semidefinite Relaxation of Quadratic Optimization Problems," IEEE Signal Processing Magazine, vol. 27, no. 3, pp. 20-34, May 2010.

[21] L. Demanet and P. Hand, "Stable optimizationless recovery from phaseless linear measurements," 2013.

[22] CVX Research, Inc. CVX: Matlab software for disciplined convex programming, version 2.0 beta. http://cvxr.com/cvx, September 2012.

[23] T. Isernia, O.M. Bucci, N. Fiorentino, "Shaped beam antenna synthesis problems: feasibility criteria and new strategies," Journal of elect. Waves Appl., vol. 12, pp. 103-138, 1998.

[24] M.H. Hayes, 'The reconstruction of a multidimensional sequence from the phase or magnitude of its Fourier transform," IEEE Trans. Acoust. Speech Sig. Proc., vol. 30, no. 2, pp. 140-154, April 1982.

[25] M.D. Migliore and D. Pinchera, "Phase-transition behavior in array diagnosis using sparse recovery techniques," Proc. of the EuCAP, 2012.

[26] O. Raz, N. Dudovich and B. Nadler, "Vectorial Phase Retrieval of 1-D Signals," IEEE trans. on Signal Processing, vol. 61, no. 7, pp. 16321643, April 2013.

[27] E. Osherovich, M. Zibulevsky, and I. Yavneh, "Phase Retrieval Combined With Digital Holography", 2012. [Online]. Available: http://arxiv.org/pdf/1203.0853v1.pdf

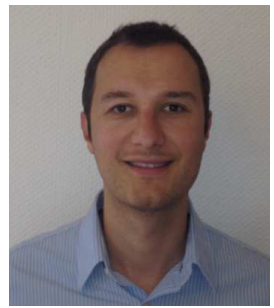

Benjamin Fuchs (S'06-M'08) received the M.S. and electrical engineering degrees in 2004 from the National Institute of Applied Science of Rennes, France. He received the Ph.D. degree in 2007 from the University of Rennes 1, France, and was during that period a visiting scholar at the University of Colorado at Boulder, USA. In 2009, he joined the Institute of Electronics and Telecommunications of Rennes (IETR) as a researcher at the Centre National de la Recherche Scientifique (CNRS). He has spent three years (2008 as postdoctoral research fellow and 2011-2012 on leave from CNRS) at the Swiss Federal Institute of Technology of Lausanne (EPFL) in Switzerland.

His research interests include millimeter-wave antennas, focusing devices (lens antennas) and array synthesis methods. 


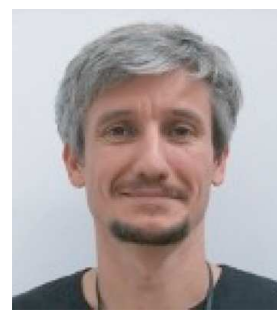

Laurent Le Coq received the electronic engineering and radiocommunications degree and the french DEA degree (M.Sc.) in electronics in 1995 and the $\mathrm{Ph}$. D. in 1999 from the National Institute of Applied Science (INSA), Rennes, France. In 1999, he joined IETR (Institute of Electronics and Telecommunications of Rennes), University of Rennes 1 , as a research lab engineer, where he is responsible of the measurement technical facilities up to $110 \mathrm{GHz}$, and of the prototyping service.

His activities in antenna measurements and development of related procedures involved him in more than 30 research contracts of national or european interest. He is author and co-author of more than 35 journal papers and 35 papers in conference proceedings. 